\title{
Processing fluency affects subjective claims of recollection
}

\author{
Brian P. Kurilla ANd Deanne L. Westerman \\ State University of New York, Binghamton, New York
}

\begin{abstract}
Previous studies that have used the remember-know paradigm to investigate subjective awareness in memory have shown that fluency manipulations have an impact on "know" responses but not on "remember" responses (e.g., Rajaram, 1993), a finding typically accounted for by invoking inferential processing in judgments of familiarity but not of recollection. However, in light of several researchers' criticisms of this procedure, as well as findings documenting the influence of processing fluency on various subjective judgments, the present study was conducted in order to investigate whether judgments of recollection might also be subject to inferential processes and not solely the product of conscious retrieval. When the standard remember-know procedure was used (Experiment 1), manipulations of perceptual fluency increased "know" responses but had no effect on "remember" responses, replicating previous findings. However, when an independent ratings method was employed (Higham \& Vokey, 2004), manipulations of perceptual fluency (Experiment 2) and conceptual fluency (Experiment 3) reliably increased claims of both familiarity and recollection, suggesting that the conclusion that fluency affects only "know" responses may be an artifact of the standard remember-know procedure.
\end{abstract}

The seminal work of Tversky and Kahneman (1974) demonstrated how our everyday decision making is guided by the use of heuristics. In recent years, this line of thinking has been applied to the study of human memory, and it is now well established that a number of heuristics are used in making mnemonic judgments, such as recognition. One of the most frequently investigated heuristics in memory is the fluency heuristic (for a review, see Kelley \& Rhodes, 2002). Beginning with the work of Jacoby and colleagues (e.g., Jacoby \& Dallas, 1981; Jacoby \& Whitehouse, 1989), the relative speed and ease with which stimulus information is processed has been implicated as a contributing factor in item recognition, with greater claims of recognition given to items that are processed fluently than to those that are processed less fluently. The general idea is as follows: Because repeated presentations of a stimulus generally result in facilitated processing of that stimulus (i.e., the phenomenon of repetition priming), fluent processing is interpreted as a sign of prior experience and therefore serves as a cue for recognition.

Importantly, however, processing fluency may arise from sources other than repetition, and as such, it has been shown to influence a number of nonmemorial judgments as well. For instance, fluency has been shown to affect judgments concerning stimulus duration (see, e.g., Masson \& Caldwell, 1998), brightness (e.g., Mandler, Nakamura, \& Van Zandt, 1987), likability (e.g., Reber, Winkielman, \& Schwarz, 1998; Reber, Zimmermann, \& Wurtz, 2004), truthfulness (e.g., Hasher, Attig, \& Alba, 1981; Hasher, Goldstein, \& Toppino, 1977), feelings of knowing (e.g., Begg, Robert- son, Gruppuso, Anas, \& Needham, 1996; Westerman \& Greene, 1999), and even fame (e.g., Jacoby, Kelley, Brown, \& Jasechko, 1989; Jacoby, Woloshyn, \& Kelley, 1989), with the effects of fluency determined in these cases by individuals' interpretations of the source of fluent processing (i.e., whether fluent processing is seen as being due to past experience, inherent properties of the stimulus, or some other source entirely; Jacoby, Kelley, \& Dywan, 1989; Jacoby \& Whitehouse, 1989; Whittlesea, 1993).

The findings described previously suggest that many of our everyday decisions - both memorial and nonmemorial - are influenced by our interpretation of factors that could plausibly give rise to fluent stimulus processing (e.g., prior exposure vs. stimulus attributes). However, within the realm of recognition memory, it is commonly accepted that recognition decisions can be based on either familiarity or recollection (see, e.g., Atkinson \& Juola, 1973; Jacoby, 1991; Mandler, 1980; Tulving, 1985; Yonelinas, 1994, 2002), and that only the former is reliably influenced by manipulations of fluency. Dualprocess theories of recognition memory generally contend that feelings of familiarity arise from a fast and effortless process that is largely inferential in nature, and that feelings of recollection result from a slower, more effortful process that is used for retrieving episodic and contextual detail that is encoded during study. Over the past two decades, this class of theories has received substantial empirical support (e.g., Gardiner, 1988; Gardiner, Gregg, \& Karayianni, 2006; Gardiner, Java, \& Richardson-Klavehn, 1996; Gruppuso, Lindsay, \& Kelley, 1997; Hintzman \& 
Curran, 1994; Jacoby, 1991; Rajaram, 1993, 1996), and the notion that recognition can be supported by familiarity or recollection appears to be well founded. What remains unclear, however, is whether subjective feelings of familiarity and recollection actually map directly onto separate processes that are characterized by inference and retrieval, respectively. Viewing claims of familiarity and recollection as subjective judgments (like judgments of duration, likability, fame, and so forth), it is conceivable that both might be influenced by variations in fluency.

The goal of the present study was to investigate the notion that fluency affects only impressions of familiarity and to determine whether it might be the case that under some circumstances, recollection may be an inference that is susceptible- like the many others mentioned previously - to fluency manipulations. In order to investigate this possibility, one must consider judgments that are typically thought to rely on strict retrieval mechanisms, but at the same time to reflect some degree of subjectivity. One clear example of such a decision-and the one with which we are principally concerned in the present investigation - is the "remember" response as it is conceived in the remember-know paradigm (Gardiner, 1988).

In the remember-know paradigm, participants are required to introspect about the nature of their memorial experience during a recognition memory test and to categorize each positive recognition response as one of either remembering or knowing, which-according to Tulving (1985) - requires access to qualitatively different types of retrieved information (e.g., episodic vs. semantic, respectively). Under typical instructions, participants are asked to provide a "remember" response if they can retrieve some thought or association that directly links a test item to the prior study phase, but to provide a "know" response if they feel confident that the item was studied but they cannot retrieve specific contextual information. In a study concerned with investigating the nature of subjective states of memory awareness, Rajaram (1993) used the remember-know paradigm in conjunction with a priming procedure that was developed by Jacoby and Whitehouse (1989), in which half of all items on a recognition test were preceded by briefly presented matching primes. Arguing that primes serve to enhance the perceptual fluency of subsequent test items, Rajaram (1993) found that in comparison with unprimed items, primed items were more likely to receive positive recognition responses, a finding previously reported by Jacoby and Whitehouse and since replicated by several researchers (e.g., Westerman, 2001; Westerman, Lloyd, \& Miller, 2002). More importantly, however, Rajaram (1993) also found that primed items were more likely to receive "know" responses, but that they were no more likely to receive "remember" responses than were unprimed items.

Although these findings seem to provide an example of a class of subjective judgments that are unaffected by processing fluency and provide support for the perspective that feelings of familiarity but not feelings of recollection are partly determined by inference, note that some researchers disagree with this distinction, arguing that it overlooks important similarities between familiarity and recollection. For instance, Whittlesea and colleagues
(Leboe \& Whittlesea, 2002; Whittlesea, 2002) agreed with the general notion that recognition performance can be supported by either familiarity or recollection, but they disagreed with the contention that familiarity and recollection are invariably tied to separate underlying processes that involve the retrieval of qualitatively distinct information. They also disagreed with the idea that feelings of recollection arise solely as a by-product of pure retrieval. These ideas are embodied in Whittlesea's $(1997,2002)$ framework for selective construction and preservation of experience (SCAPE), which asserts that both feelings of familiarity and feelings of recollection are products of inference and attribution of current stimulus processing to a previously encountered source.

There is already some support for the idea of an inferential basis for feelings of recollection. A study conducted by Bodner and Lindsay (2003) used the remember-know paradigm to examine contextual influences upon subjective states of awareness. In their study, participants studied two separate lists of words under different encoding conditions - one group studied one list in a medium level of processing (LOP) and the other in a shallow LOP, and a second group studied one list in a medium LOP and the other in a deep LOP. During the following recognition memory test, participants from both groups were presented with a list of words comprised of items from their respective study lists mixed with novel lures, and they were asked to provide judgments of remembering and knowing. The most important finding for present purposes was that comparing across groups, medium LOP items in the medium-with-shallow test context received significantly more "remember" responses than did medium LOP items in the medium-with-deep test context. However, claims of remembering were equivalent when the test list was comprised entirely of medium LOP items mixed with novel lures, thereby ruling out the possibility that differences in encoding might have been responsible (Bodner \& Lindsay, 2003, Experiment 2). Still more evidence comes from a recent study by Rotello, Macmillan, Reeder, and Wong (2005) in which they found that "remember" responses in the remember-know paradigm are influenced by the type of instructions that participants receive concerning the manner of responding. Specifically, Rotello et al. (2005) found that the number of "remember" responses assigned to targets and lures was reduced under instructional conditions in which participants were required to provide rationales for remembered items in comparison with conditions in which no such rationales were required (Rotello et al., 2005, Experiment 1), indicating that the "remember" response is susceptible to response bias (also see Hirshman \& Henzler, 1998, for a demonstration of bias in the "remember" response). Thus, even though the previously mentioned studies (e.g., Bodner \& Lindsay, 2003; Rotello et al., 2005; Whittlesea, 2002) differ at the procedural level, their general findings and conclusions converge on the idea that remembering is a subjective memorial experience that is influenced by factors beyond mere retrieval of episodic and contextual detail.

This reasoning is also consistent with models of recognition memory that see recollection as being based 
on a graded, continuous variable (see, e.g., Reder et al., 2000; Rotello, Macmillan, \& Reeder, 2004; Wixted \& Stretch, 2004) rather than an all-or-none threshold-like process (e.g., Yonelinas, 1994). If true, then the standard remember-know paradigm ignores this and forces participants to artificially dichotomize their subjective experiences. Although such criticisms have most often been seen as a problem for "know" responses (e.g., Lindsay \& Kelley, 1996; Yonelinas \& Jacoby, 1995), it is conceivable that this might also affect people's "remember" responses. For instance, forcing participants to choose between one of two dichotomous response categories might lead them to occasionally classify relatively moderate and weak experiences of remembering as knowing.

The present study sought to expand on the notion that subjective feelings of recollection are partly inferential in nature by demonstrating that claims of recollection can sometimes be dissociated from a straightforward retrieval process, which - from the perspective of most dual-process theories - is the hallmark characteristic of recollection. Furthermore, the present investigation sought corroborating evidence for newer models of memory that emphasize graded (and false) recollection (e.g., Whittlesea, 1997, 2002; Wixted \& Stretch, 2004) by demonstrating that claims of recollection, like claims of familiarity and other subjective judgments, are influenced by manipulations of processing fluency. In Experiment 1, we attempted to replicate the findings of Rajaram (1993) using the standard remember-know paradigm and the perceptual priming procedure that was developed by Jacoby and Whitehouse (1989). In Experiments 2 and 3, we investigated the possibility that her findings might be an artifact of the remember-know paradigm itself and, as such, not an accurate depiction of the influence of processing fluency on subjective memorial experiences. Thus, in Experiments 2 and 3, we used an independent ratings methodology (Higham and Vokey, 2004) that allows for independent and graded assessments of the extent to which every test item is both familiar and recollected. Furthermore, rather than manipulating perceptual fluency as in Experiments 1 and 2, in Experiment 3, we extended our investigation to include a manipulation of conceptual fluency.

\section{EXPERIMENT 1}

In Experiment 1, we intended to replicate the findings of Rajaram (1993), which showed that when the standard remember-know paradigm is employed, manipulations of perceptual fluency primarily influence claims of knowing rather than claims of remembering. From a dual-process perspective, such a pattern of results is consistent with and easily explained by the constructive and attributional nature of the familiarity process. Recollection, on the other hand, which is typically seen as resulting from an active process of search-and-retrieval from memory, would not be expected to be influenced by such a manipulation. However, as was alluded to earlier, a replication of the findings of Rajaram (1993) need not be taken as solid evidence that feelings of familiarity but not feelings of recollection are influenced by processing fluency. This pattern of results could be an artifact of the remember-know procedure itself. In keeping with the methodology of Rajaram (1993), participants were initially presented with a study list of words in anticipation of an upcoming memory test. During the subsequent recognition memory test, half of the test words were preceded by a brief matching prime, which served as a manipulation of perceptual fluency. On the recognition test, participants gave a yes-no recognition response and then indicated whether recognized test items were remembered or known.

\section{Method}

Design. Experiment 1 was conducted as a 2 (test item status: old vs. new) $\times 2$ (prime type: matching vs. nonmatching) repeated measures design, with the proportion of "yes," "remember," and "know" responses to items in the test list serving as dependent variables.

Participants. Seventy students from the State University of New York at Binghamton participated in order to fulfill a course requirement. The participants were tested individually.

Materials. The stimuli consisted of 126 words that were drawn from a pool that was taken from the MRC Psycholinguistic Database (www.psy.uwa.edu.au/mrcdatabase/uwa_mrc.htm). Of these 126 words, 30 served as targets in the initial study list, which were to be presented again during the test phase, and 30 served as filler items. Additionally, six words were used as primacy and recency buffers, which occupied the first and last three serial positions in the study list for all participants. Aside from these buffers, all items in the study and test phase were randomly presented. The test list consisted of 60 words, 30 of which had been studied earlier and 30 of which were new. Furthermore, half of the items $(15$ old and 15 new) were preceded by a briefly presented matching prime and the other half were preceded by a briefly presented nonmatching prime. Counterbalancing across participants assured that each test item appeared equally as often as an old and new item, and that each test item was preceded equally as often by a matching and a nonmatching prime.

Procedure. Participants were first presented with a study list of 60 words and were instructed to pay close attention to each item in preparation for an upcoming memory test. The words on the study list were presented individually in the center of a 17 -in. color monitor at a rate of $1 \mathrm{sec}$ each and with a 500-msec interval between each presentation. After the study phase, the experimenter informed participants that they were about to take a memory test in which some words were previously studied and others were not. They were informed that each item that had been assigned a "yes" response on the general yes-no recognition task would be followed by a remember-know judgment (instructions for the remember-know distinction were taken directly from Rajaram, 1993). Participants were instructed to respond "remember" whenever they were able to retrieve some specific contextual aspect from prior study (e.g., physical appearance of the word, what they were thinking at the moment the word was initially presented, the position of the word in the study list, etc.) and to respond "know" whenever they recognized a word as one that was from the study list but for which they were unable to retrieve any specific contextual/associative details encoded during study (i.e., familiarity in the absence of recollection). As an additional check on their understanding of the distinction between these subjective experiences, prior to the recognition test, participants were presented with a pretest of four scenarios that provided idealistic examples of remembering and knowing (two scenarios depicted instances of remembering and two depicted instances of knowing). Participants were instructed to read each scenario and indicate by keypress the memorial experience that best characterized each event. After completion of the four scenarios, participants could ask questions and seek clarification. Then, they were prompted by the experimenter to put the distinction between remembering and knowing into their own words. Once the experimenter was satisfied with participants' descriptions, they began the recognition memory test. 
During the test, participants were presented with 60 words ( 30 old, 30 new) in serial fashion, and each word was preceded by the brief presentation of either a matching or nonmatching word. Each test trial began with a $1-\mathrm{sec}$ presentation of a plus sign serving as a fixation point in the center of the screen. This was followed by presentation of a forward mask of number signs (\#\#\#\#\#\#) that was present on the screen for $250 \mathrm{msec}$, and then a prime for $34 \mathrm{msec}$ followed by a backward mask of number signs (\#\#\#\#\#\#). Participants were not informed about the presentation of preceding primes, but they were informed about the occurrence of the number signs before each test item presentation. Participants were instructed to use these number signs as a signal that the test word was about to appear. Following offset of the backward mask, the screen was blank for $500 \mathrm{msec}$, at which point the target word was presented in the center of the screen along with the prompt "Was this word on the study list?" Participants were told to press the "y" key for items they believed to have been on the list and the " $n$ " key for items they believed not to have been on the list. If participants pressed "y," then they were asked for judgments of memorial experience. In this case, the test word remained in the center of the screen, but the prompt changed from one concerned with general recognition (yes-no judgment) to one concerned with recollection and familiarity ("remember or know?"). After the remember-know judgment was made, the screen was blank for $4 \mathrm{sec}$, and then the next experimental trial began. If participants initially pressed "n," the remember-know judgment was bypassed, and the next trial began.

\section{Results and Discussion}

A significance criterion of .05 was used to interpret all statistical tests. Participants were required to demonstrate adequate understanding of the distinction between remembering and knowing; thus, they needed to exhibit at least $75 \%$ accuracy on the pretest. Seven participants were removed from the data set for failure to reach this criterion.

In order to interpret the influence of the priming manipulation on general claims of recognition and specific claims of remembering and knowing, three separate 2 (item status: target vs. lure) $\times 2$ (prime type: matching vs. nonmatching) within-subjects ANOVAs were conducted on the proportions of "yes" responses, "remember" responses, and "know" responses. A summary of the results of these analyses can be found in Table 1. For the test of general recognition (i.e., "yes" responses), there was a main effect of item status $\left[F(1,62)=358.34, M S_{\mathrm{e}}=\right.$ $\left..03, \eta^{2}=.85\right]$, indicating that previously studied targets received a higher proportion of "yes" responses than did previously nonstudied lures. There was also a main effect of prime type $\left[F(1,62)=17.30, M S_{\mathrm{e}}=.02, \eta^{2}=.22\right]$, indicating that test items that were preceded by matching primes received a higher proportion of "yes" responses than did items that were preceded by nonmatching primes. The interaction between item status and prime type was not significant $(F<1)$.

Turning to consideration of the influence of the priming manipulation on subjective memorial experiences, analyses of the "remember" responses revealed a main effect of item status only $\left[F(1,62)=270.94, M S_{\mathrm{e}}=.02, \eta^{2}=.81\right]$, reflecting the fact that targets received a higher proportion of "remember" responses than did lures. For "remember" responses, neither the main effect of prime type nor the interaction between item status and prime type reached significance. For "know" responses, there was also a main ef- fect of item status $\left[F(1,62)=31.85, M S_{\mathrm{e}}=.02, \eta^{2}=.34\right]$, reflecting the fact that targets received a higher proportion of "know" responses than did lures. However, unlike "remember" responses, for "know" responses, the main effect of prime type was significant $\left[F(1,62)=12.77, M S_{\mathrm{e}}=.02\right.$, $\left.\eta^{2}=.17\right]$. Primed test items were more likely than unprimed test items to receive "know" responses. As was the case with "remember" responses, there was no significant interaction between item status and prime type $(F<1)$.

Researchers often argue that "know" responses that are gathered from the standard remember-know paradigm provide a biased estimate of the familiarity process because they merely reflect an estimate of familiarity in the absence of recollection. To correct for this biasing, Yonelinas and Jacoby (1995) suggested that the proportion of "know" responses be divided by 1 minus the proportion of "remember" responses. Accordingly, we divided the mean proportion of "know" responses for all participants (for both matching and nonmatching prime conditions) by 1 minus the proportion of "remember" responses and then subjected these adjusted "know" responses to a 2 (item status) $\times 2$ (prime type) repeated measures ANOVA. The results of this analysis (displayed in the lower portion of Table 1) showed the same pattern as the unadjusted "know" responses: a main effect of item status $[F(1,62)=$ $\left.145.99, M S_{\mathrm{e}}=.03, \eta^{2}=.70\right]$, a main effect of prime type $\left[F(1,62)=14.89, M S_{\mathrm{e}}=.03, \eta^{2}=.19\right]$, and no interaction between item status and prime type $(F<1)$.

Our results replicated the pattern that was reported by Rajaram (1993). Primed items received a higher proportion of "yes" responses than did unprimed items on a yesno recognition test, and more importantly, this priming effect was primarily observed in "know" responses, with little influence on "remember" responses. This finding is consistent with dual-process theories of recognition, which contend that feelings of familiarity and recollection are distinct in terms of their underlying cognitive processes. From this perspective, feelings of familiarity can be seen as arising from attribution of unexpectedly high levels of

Table 1

Mean Proportions, With Standard Errors, of "Yes," "Remember," "Know," and "Adjusted Know" Responses As a Function of Item Status and Prime Type for Experiment 1

\begin{tabular}{llllllr}
\hline & \multicolumn{4}{c}{ Text Condition } & \\
\cline { 2 - 3 } Response Type and & \multicolumn{2}{c}{ Primed } & & Not Primed & Priming \\
\cline { 2 - 3 } \cline { 5 - 6 } Item Status & $M$ & $S E$ & & $M$ & $S E$ & Effect \\
\hline Y/N Recognition & .46 & .02 & .39 & .02 & .07 \\
Target & .67 & .02 & .61 & .03 & .06 \\
Lure & .26 & .02 & .18 & .02 & .08 \\
Remember & .20 & .01 & .20 & .01 & .00 \\
Target & .35 & .02 & .36 & .02 & -.01 \\
Lure & .06 & .01 & .03 & .01 & .03 \\
Know & .26 & .02 & .20 & .01 & .06 \\
Target & .31 & .02 & .24 & .02 & .07 \\
Lure & .21 & .02 & .15 & .02 & .06 \\
Adjusted Know & .36 & .02 & .27 & .02 & .02 \\
Target & .49 & .03 & .39 & .03 & .10 \\
Lure & .22 & .02 & .16 & .02 & .06 \\
\hline
\end{tabular}


perceptual fluency to repeated exposure of a stimulus, even though this enhanced fluency is actually due to the brief presentation of a masked prime. Furthermore, recollection is seen as arising strictly from a retrieval process whereby individuals attempt to retrieve contextual and associative details that link a stimulus with prior presentation during the study phase. As such, there is no reason why a prime, which serves to facilitate current stimulus processing, should have much of an impact on claims of recollection.

Still, this pattern of findings does not necessarily require such an explanation. It could be the case that participants are forced into this pattern by the dichotomous nature of the remember-know task. Indeed, the assumption that is implicitly conveyed to participants during this task is that one either remembers or does not remember and either knows or does not know. Thus, if recollection is in actuality a graded process (see, e.g., Reder et al., 2000; Rotello et al., 2004; Wixted \& Stretch, 2004), then relatively weak experiences of remembering might occasionally be categorized as instances of knowing. For this reason, in Experiment 2, we employed a procedure that required separate and independent ratings of familiarity and recollection (Higham \& Vokey, 2004), believing that this might be more sensitive to the graded nature of recollection and therefore allow for fluency effects to be found in these judgments.

\section{EXPERIMENT 2}

In Experiment 2, we used an independent ratings method that was developed by Higham and Vokey (2004) to address limitations of the standard remember-know paradigm. ${ }^{1}$ This procedure, which requires participants to provide separate and independent ratings of familiarity and recollection for every word in a recognition memory test, avoids the previously mentioned response dichotomy (one either remembers or does not and one either knows or does not) and resultant dependence between responses, either of which could potentially obscure the effects of variables on subjective memorial experience. Thus, the question of interest in Experiment 2 is whether the same pattern of results that was found in Experiment 1 (an elevation in claims of familiarity with no change in claims of recollection) will be observed when participants are allowed to provide independent and graded ratings of subjective memorial experience for every test word.

The procedure of Experiment 2 was very similar to that of Experiment 1, with the exception of the response phase. Instead of using the standard remember-know procedure, in Experiment 2, we used an independent scales methodology (Higham \& Vokey, 2004). Participants were again given an initial list of words to study and then tested for recognition, and each test word was again preceded by a brief presentation of either a matching or nonmatching perceptual prime. However, unlike in Experiment 1, we did not solicit general yes-no recognition. Rather, participants were merely asked to provide two ratings for each test item, one to indicate the extent to which a word could be "recollected" and another to indicate the extent to which a word seemed "familiar." Given the predictions of models of memory that dispute the threshold-like nature of recollection (see, e.g., Reder et al., 2000; Rotello et al., 2004; Whittlesea, 1997, 2002; Wixted \& Stretch, 2004) and the large body of findings that demonstrate fluency effects for subjective judgments (e.g., Begg et al., 1996; Hasher et al., 1981; Hasher et al., 1977; Jacoby, Kelley, et al., 1989; Jacoby, Woloshyn, \& Kelley, 1989; Mandler et al., 1987; Masson \& Caldwell, 1998; Reber et al., 1998; Reber et al., 2004; Westerman \& Greene, 1999), we anticipated that the presence of a prime would enhance claims of recollection when responses were free to vary independently and to be expressed along a graded continuum. Critically, such influence of a prime upon claims of recollection would stand in opposition to any dual-process account of recognition memory that characterizes claims of recollection as being based entirely on retrieval of episodic information.

\section{Method}

Design. Experiment 2 was conducted as a 2 (item status: target vs. lure) $\times 2$ (prime type: matching vs. nonmatching) $\times 2$ (response scale: familiarity vs. recollection) repeated measures design, with mean ratings on the recollection and familiarity scales serving as dependent variables.

Participants. Forty-four students from the State University of New York at Binghamton participated to fulfill a course requirement. The participants were tested individually.

Materials and Procedure. The materials and procedure of Experiment 2 were identical to those of Experiment 1, with one exception. Instead of using the standard remember-know procedure, participants were required to rate each test item on two separate scales of 1 to 4 , with one reflecting the extent to which words could be "recollected" and the other reflecting the extent to which words seemed "familiar" from the study phase (where 1 was definitely no and 4 was definitely yes). The order of the ratings was counterbalanced across participants so that half of the participants rated recollection first and the other half rated familiarity first. Prior to the test, participants progressed through a series of instructions on the computer that clarified at length the relevant distinctions between familiarity and recollection (taken directly from Higham \& Vokey, 2004). Recollection was described as the ability to become consciously aware again of some aspect or aspects of what was previously experienced, and that it is typically characterized by retrieval of such episodic detail as physical appearance and thoughts/associations that occurred during initial presentation. Conversely, familiarity was described as a vague feeling of having seen an item before with or without conscious recollection of episodic detail. Furthermore, the instructions emphasized independence between feelings of recollection and feelings of familiarity, stating that it is possible to have memories characterized by high recollection and low familiarity (HR-LF), low recollection and high familiarity (LR-HF), high recollection and high familiarity (HR-HF), or low recollection and low familiarity (LR-LF). As in the previous experiment, participants were presented with a pretest of four scenarios that provided idealistic examples of the four possible combinations of memorial experience. Participants were instructed to read each scenario and to indicate by keypress the memorial experience that best characterized each event (e.g., HR-LF, LR-HF, HR-HF, or LR-LF). Afterward, participants could ask questions or seek clarification before they were prompted by the experimenter to put the distinction between recollection and familiarity into their own words. Once the experimenter was satisfied with the participants' descriptions, they moved on to recognition memory test.

\section{Results and Discussion}

Prior to conducting any statistical analyses on the data from Experiment 2, each participant's performance on the 
four-question pretest was examined in order to ensure adequate understanding of the relevant distinctions between feelings of recollection and feelings of familiarity. As in the previous experiment, participants were required to exhibit at least $75 \%$ accuracy on the pretest. ${ }^{2}$ Four participants were removed from the data set for failure to reach this criterion.

Turning to the consideration of participants' reported subjective experiences for test words, mean ratings were analyzed with a 2 (item status: target vs. lure) $\times 2$ (prime type: matching vs. nonmatching) $\times 2$ (response scale: recollection vs. familiarity) within-subjects ANOVA. A summary of the results can be found in Table 2. The analysis revealed a main effect of status $\left[F(1,39)=239.40, M S_{\mathrm{e}}=\right.$ $.27, \eta^{2}=.86$, with targets receiving higher mean ratings than lures]; a main effect of prime type $[F(1,39)=9.16$, $M S_{\mathrm{e}}=.17, \eta^{2}=.19$, with items preceded by matching primes receiving higher mean ratings than items preceded by nonmatching primes]; and a main effect of response scale $\left[F(1,39)=41.21, M S_{\mathrm{e}}=.44, \eta^{2}=.52\right.$, with mean familiarity ratings exceeding mean recollection ratings]. The only interaction to approach significance was between status and prime type $\left[F(1,39)=5.13, M S_{\mathrm{e}}=.10, p<\right.$ $\left..057, \eta^{2}=.09\right]$, reflecting the fact that priming effects were larger for nonstudied lures than for studied targets. Critically, there was no significant interaction between prime type and scale $\left[F(1,39)=2.86, M S_{\mathrm{e}}=.02, p=.10\right]$. Separate planned comparisons carried out on the mean ratings for the recollection and familiarity scales showed that fluency affected both scales; higher ratings were given to primed test words than to unprimed test words for both familiarity and recollection $[t(39)=2.44, S E=.04$ for the familiarity scale, and $t(39)=3.17, S E=.05$ for the recollection scale]. Finally, in order to facilitate comparison with the overall recognition data from Experiment 1, we estimated the hit and false alarm rates by examining the proportion of trials on which participants provided either a 3 or a 4 rating on either scale (targets, primed $=.70$ vs. unprimed $=.67$; lures, primed $=.44 \mathrm{vs}$. unprimed $=.36$ ) .

The present findings stand in contrast to the results of Experiment 1 and the original findings of Rajaram (1993), which both found that a priming manipulation affected "know" responses but not "remember" responses in the standard remember-know procedure and, therefore, lend

Table 2

Mean Ratings, With Standard Errors, on the Recollection and Familiarity Scales As a Function of Item Status and Prime Type for Experiment 2

\begin{tabular}{|c|c|c|c|c|c|}
\hline \multirow{3}{*}{$\begin{array}{l}\text { Response Scale } \\
\text { and Item Status }\end{array}$} & \multicolumn{4}{|c|}{ Test Condition } & \multirow{3}{*}{$\begin{array}{l}\text { Priming } \\
\text { Effect }\end{array}$} \\
\hline & \multicolumn{2}{|c|}{ Primed } & \multicolumn{2}{|c|}{ Not Primed } & \\
\hline & $M$ & $S E$ & $M$ & $S E$ & \\
\hline \multicolumn{6}{|l|}{ Recollection Scale } \\
\hline Target & 2.74 & .08 & 2.64 & .09 & .10 \\
\hline Lure & 1.84 & .09 & 1.60 & .06 & .24 \\
\hline Overall & 2.29 & .07 & 2.12 & .07 & .17 \\
\hline \multicolumn{6}{|l|}{ Familiarity Scale } \\
\hline Target & 3.11 & .07 & 3.08 & .08 & .03 \\
\hline Lure & 2.37 & .12 & 2.17 & .12 & .20 \\
\hline Overall & 2.74 & .09 & 2.63 & .10 & .12 \\
\hline
\end{tabular}

some support to the idea that these prior findings might be an artifact of the paradigm itself. Nonetheless, given the seemingly widespread acceptance of the notion that feelings of familiarity but not feelings of recollection are based on inference, a replication of the results from Experiment 2 would seem in order. Therefore, Experiment 3 was conducted partly as an attempt to replicate the results from Experiment 2. However, it was also conducted to see whether these results are limited to manipulations of perceptual fluency or whether they might extend to more conceptually driven sources of processing fluency as well.

\section{EXPERIMENT 3}

In Experiment 3, we sought converging evidence for the role of inference in feelings of recollection by investigating whether similar elevations in ratings of recollection emerge with a more conceptually driven manipulation of processing fluency. Like perceptual fluency, conceptual fluency has typically been discussed in terms of its influence on feelings of familiarity (see, e.g., Whittlesea, 1993; Whittlesea \& Williams, 2001b), with little attention paid to its potential influence on feelings of recollection. However, as we have shown in Experiment 2, when using a method that requires ratings of memorial experience, perceptual fluency can have a reliable influence on subjective feelings of recollection. If such an effect is also found with a manipulation of conceptual fluency, this would further weaken the argument that feelings of recollection are strictly the by-product of retrieved episodic information, and it would provide converging evidence for the idea that inference plays a role in such judgments.

The procedure used in Experiment 3 was similar to that used in Experiment 2. The two experiments differed only with respect to the type of information that was presented prior to the onset of each test word. Instead of matching and nonmatching primes, test words in the present experiment were preceded by either predictive or nonpredictive sentence stems in order to manipulate conceptual fluency. Several researchers have employed this procedure as a means of facilitating conceptual processing of test stimuli, and research has reliably shown that items preceded by predictive stems generally receive a higher proportion of positive recognition responses than do items preceded by nonpredictive sentence stems (e.g., Miller, Lloyd, \& Westerman, 2007; Whittlesea, 1993, 2002; Whittlesea \& Wixted, 2001b). That being the case, the most important question for the present purposes is whether participants will interpret the perceived facilitation in the processing of the conceptual/semantic properties of the test stimuli as a sign that items are familiar, recollected, or both.

Currently, there is some evidence to suggest that conceptual fluency will influence subjective feelings of recollection. Whittlesea (2002) conducted a study in which stimuli were preceded by predictive or nonpredictive sentence stems either during study and test or during test alone and found that in both cases, enhanced conceptual fluency was interpreted as a sign of greater remembering (i.e., recollection) but not greater familiarity. However, because Whittlesea (2002) employed a procedure that 
merely required participants to report one of two dichotomous memorial responses (e.g., actually recall vs. feels familiar), it is unknown as of yet whether this finding will hold when subjective memorial judgments are free to be expressed in graded fashion. Given the results of Experiment 2, we expected that the presentation of predictive sentence stems would lead to elevated ratings on both the recollection and familiarity scales.

\section{Method}

Design. Experiment 3 was conducted as a 2 (item status: target vs. lure) $\times 2$ (sentence stem type: predictive vs. nonpredictive) $\times 2$ (response scale: familiarity vs. recollection) repeated measures design, with mean ratings on the familiarity and recollection scales serving as dependent variables.

Participants. Sixty-eight students from the State University of New York at Binghamton participated in order to fulfill a course requirement. The participants were tested individually.

Materials and Procedure. The materials and procedure of Experiment 3 were identical to those in Experiment 2 . The only difference was that test items in Experiment 3 were preceded by either predictive or nonpredictive sentence stems instead of matching or nonmatching primes. Most of the sentences were from the pool that was used by Whittlesea and Williams (2001a), but we supplemented the Whittlesea and Williams (2001a) list with similar sentences that were constructed in our lab. Both predictive and nonpredictive sentence stems were generated for every test word, but for any given participant, half of the items ( 15 old and 15 new) were preceded by predictive sentence stems and the other half were preceded by nonpredictive sentence stems. Each test word served as the terminal item in the sentence and always completed it coherently. For example, the test item PERMIT could be preceded by the stem You can only drive after obtaining a learner's ... (predictive) or I had to go to the other room to get my ... (nonpredictive). Counterbalancing across participants was employed so that all items were equally likely to be preceded by either a predictive or a nonpredictive sentence stem.

At the start of each test trial, either a predictive or a nonpredictive sentence stem was presented in the center of the computer screen and remained on the screen until the participant completed reading it and pressed the space bar. After the participant did so, the screen was blank for $250 \mathrm{msec}$ until the terminal test word (PERMIT) was presented in capital letters in the center of the screen along with the prompt to rate memory awareness (i.e., recollection or familiarity depending upon the counterbalancing condition). After making their rating on the first scale, participants were immediately asked for a rating on the remaining scale.

\section{Results and Discussion}

Prior to conducting any statistical analyses on the data from Experiment 3, each participant's performance on the pretest was examined to ensure understanding of the distinction between feelings of recollection and feelings of familiarity. As in the previous experiments, participants were required to have achieved at least $75 \%$ accuracy on the pretest in order to be included in subsequent statistical analyses. Twelve participants were removed from the data set for failure to reach this criterion.

A summary of the data can be found in Table 3 . A 2 (item status: old vs. new) $\times 2$ (sentence stem type: predictive vs. nonpredictive) $\times 2$ (scale: recollection vs. familiarity) within-subjects ANOVA revealed main effects of item status $\left[F(1,55)=147.91, M S_{\mathrm{e}}=.68, \eta^{2}=\right.$ .73 , with old items receiving higher mean ratings than new items $]$, sentence type $\left[F(1,55)=11.44, M S_{\mathrm{e}}=.10, \eta^{2}=\right.$
.17 , with items preceded by predictive sentence stems receiving higher mean ratings than items preceded by nonpredictive sentence stems $]$, and scale $[F(1,55)=15.00$, $M S_{\mathrm{e}}=.50, \eta^{2}=.21$, with higher mean ratings on the familiarity scale than on the recollection scale]. Additionally, there was a significant interaction between status and scale $\left[F(1,55)=4.98, M S_{\mathrm{e}}=.10, \eta^{2}=.08\right]$, reflecting somewhat better discrimination between old and new items when rating recollection than when rating familiarity. Notably, as in Experiment 2, there was no significant interaction between sentence type and response scale or between any other factors. Separate planned comparisons carried out on the mean ratings for the recollection and familiarity scales showed that higher ratings were given to the predicted test words than to the nonpredicted test words on both scales $[t(55)=3.42, S E=.03$ for the familiarity scale, and $t(55)=2.67, S E=.02$ for the recollection scale]. Finally, as in Experiment 2, we estimated the hit and false alarm rates by examining the proportion of trials on which participants provided a rating of 3 or 4 on either scale (targets, primed $=.70$ vs. unprimed $=.68$; lures, primed $=.39$ vs. unprimed $=.31$ ).

The present findings are in agreement with the results of Experiment 2, which showed that enhanced perceptual fluency of test items led to elevated ratings of both familiarity and recollection, and they are problematic for any dual-process account of recognition that tries to explain subjective feelings of recollection as purely a by-product of retrieved episodic information. For such an account to hold, one would have to presume that episodic detail is retrieved more efficiently when stimuli are preceded by predictive sentence stems than by nonpredictive sentence stems (and when preceded by matching perceptual primes than by nonmatching perceptual primes), which would be a plausible explanation if it were not for the fact that in this and the previous experiment, the largest priming effects emerged in new items, for which there was no memory trace and therefore no episodic information to be retrieved.

\section{GENERAL DISCUSSION}

The results of the present experiments indicate that enhanced processing fluency leads individuals to claim

Table 3

Mean Ratings, With Standard Errors, on the Recollection and Familiarity Scales As a Function of Item Status and Sentence Type for Experiment 3

Test Condition

\begin{tabular}{|c|c|c|c|c|c|}
\hline \multirow{3}{*}{$\begin{array}{l}\text { Response Scale } \\
\text { and Item Status }\end{array}$} & & \multirow{3}{*}{$\begin{array}{r}\text { Priming } \\
\text { Effect }\end{array}$} \\
\hline & \multicolumn{2}{|c|}{ Predictive } & \multicolumn{2}{|c|}{ Nonpredictive } & \\
\hline & $M$ & $S E$ & $M$ & $S E$ & \\
\hline \multicolumn{6}{|l|}{ Recollection Scale } \\
\hline Target & 2.86 & .06 & 2.82 & .06 & .04 \\
\hline Lure & 1.88 & .07 & 1.77 & .08 & .11 \\
\hline Overall & 2.37 & .05 & 2.29 & .05 & .08 \\
\hline \multicolumn{6}{|l|}{ Familiarity Scale } \\
\hline Target & 3.07 & .07 & 3.00 & .08 & .07 \\
\hline Lure & 2.23 & .08 & 2.07 & .07 & .16 \\
\hline Overall & 2.65 & .06 & 2.53 & .06 & .12 \\
\hline
\end{tabular}


that items are both more familiar and more completely recollected, and that this pattern of results emerges only when a procedure that allows for independent and graded ratings of memorial experience is used. When the standard remember-know procedure was used, enhancing the perceptual fluency of test words led to an increase in the proportion of "know" responses, but had little effect on the proportion of "remember" responses (Experiment 1). This pattern of results seems consistent with the proposal by recent models of recognition memory (see, e.g., Wixted, 2007; Wixted \& Stretch, 2004) that recollection is graded and based on a continuous distribution of evidence (much like familiarity) rather than on a discrete threshold (e.g., Yonelinas, 1994). If recollection is truly based on a continuous variable, the standard remember-know paradigm does not capture this and instead implicitly suggests to participants that remembering is all or none. This reasoning opens up the possibility that the pattern of results observed in Experiment 1 - and furthermore, those observed by Rajaram (1993) - are artifacts of the remember-know procedure, and therefore do not accurately depict the influence of processing fluency on subjective claims of recollection and familiarity.

These findings cannot easily be explained by models of recognition memory that regard recollection as being based purely on an all-or-none threshold-like retrieval process, because these models typically do not allow for graded "remember" responses and because they have no easy way to account for false claims of recollection (see, e.g., Yonelinas, 1994). In Experiments 2 and 3 of the present study, we observed the largest numerical priming effects for both the familiarity and recollection scales in the new items. These findings are even problematic for some models that agree with the idea that recollection is a graded process. For instance, even though Reder et al.'s (2000; Cary \& Reder, 2003) source of activation confusion (SAC) model conceptualizes recollection as being based on a continuous distribution of activation values, it nonetheless asserts that subjective judgments of recollection should be reserved for only those cases in which a word node is linked with and receives activation from an encoding-event node. Because this is only the case for words that were originally studied in the experimental context, SAC does not predict the occurrence of much false recollection. More problematic, however-at least within the context of the present study - is the fact that SAC cannot account for systematic variation within these false claims without appealing to the spread of activation among related items, only some of which are linked with an encoding-event node. Because no attempts were made in the present study to use related stimuli, this explanation for fluency affecting subjective claims of recollection does not seem viable.

The present findings do lend support for the idea that feelings of familiarity and recollection are both inferential and graded in nature. This is consistent with the broad theoretical notions of Whittlesea's $(1997,2002)$ SCAPE framework. According to the SCAPE framework, the mere retrieval of stimulus information - in the absence of a developed subjective attitude toward retrieval (consider- ing and evaluating the possible reasons why information presently comes to mind) - does not constitute a true instance of remembering. For remembering to take place, it is necessary for individuals not only to retrieve stimulus information but also to interpret the information that comes to mind as being entirely due to past experience. Whittlesea (2002) noted that if participants are given a study list comprised of strong semantic associates (e.g., bread-sandwich, onion-soup, and so forth) and are later asked whether onion was previously studied, it is quite likely that they will be able to generate soup in response. However, this act of retrieval is unlikely to be taken as a sign of remembering unless one thinks that soup has come to mind as a result of past experience and not merely as a result of its being a strong semantic associate of onion. Thus, from the perspective of SCAPE, fluency apparently has an impact on subjective claims of recollection for the same reason that it has an impact on subjective claims of familiarity-individuals continuously evaluate their processing of stimuli in the external environment and make inferences regarding the source of any fluent processing that is seen as being discrepant with expectation (e.g., Whittlesea, 1993; Whittlesea \& Williams, 1998, 2000, 2001a, 2001b).

The present findings are also consistent with the twocriterion signal detection model proposed by Wixted and Stretch (2004; for a review see Wixted, 2007). According to this model, participants set two criteria along one single dimension (memory strength) during a remember-know type task. The lower criterion specifies the strength of evidence required to make a yes-no recognition judgment, and the upper criterion specifies the strength of evidence required to make a remember judgment. Items that fall above the yes-no criterion but below the remember criterion are given a "know" response. The critical feature of this model (and the one that differentiates it from other dual-process models) is that the memory strength dimension is seen as a reflection of all available evidence for recognition and, therefore, as a combination of both recollection and familiarity. As such, Wixted and Stretch's (2004) model would simply have to assume that the fluency manipulations used presently served to elevate the strength of the test word beyond the relatively strict remember criterion.

One question that remains, however, is why the effect of a prime differs depending on whether subjective claims of recollection are measured with the standard remember-know paradigm or the independent scales method. Although we have no way of knowing for sure on the basis of the results of the present study, we can speculate on several possibilities. First, it may be the case that a prime elevates the means of both the target and lure distributions on the memory strength dimension, but leaves the decision criteria relatively unaffected. This interpretation is consistent with the findings from Experiments 2 and 3, but it is not consistent with the findings of Rajaram (1993) and Experiment 1, in which "remember" responses were unaffected by the presence of a prime. In order to accommodate these findings, one would have to make the peculiar added assumption that the recollection criterion 
becomes more conservative on primed trials when using the standard remember-know paradigm (which leads to an equal proportion of "remember" responses across primed and unprimed trials), but not when using the independent scales method. Alternatively, and somewhat more plausibly, it may be the case that a priming manipulation elevates the means of the two distributions but leaves the upper tails of the distributions (the proportion of items that are already highest in confidence and therefore reported to be recollected) relatively unaffected. ${ }^{3}$ Thus, the priming manipulations used presently might not have affected "remember" responses simply because the remember criterion is too strict, which would seem consistent with our earlier contention that the remember-know paradigm implicitly, if not explicitly, suggests to participants that remembering is all or none rather than graded. If this is accurate, then it would presumably be possible on primed trials for a portion of the target and lure distributions to exceed a more liberal criterion for recollection (namely, the less confident ratings of 3 on the independent scales method); thus, it may be the introduction of an additional lax criterion that accounts for the discrepancies between the two procedures. This interpretation, although speculative, is consistent with the finding that the priming effects in the present study were almost always larger (at least numerically) for lures and therefore for items that were weaker in terms of memory strength. Furthermore, it follows from this interpretation that priming effects should be evident for the recollection scale at the liberal criterion but not at the conservative criterion. A post hoc comparison in Experiment 2 of the proportion of primed and unprimed items rated with a 3 and a 4 on the 4-point recollection scale reveals that this was indeed the case. There was a significant priming effect for items that were given a rating of 3 , but not for items that were given a rating of 4 . The proportion of primed and unprimed items that were assigned a rating of 3 on the recollection scale was .17 and .12 , respectively $[t(39)=3.13$, $S E=.02, p<.01]$. However, the proportion that was assigned a rating of 4 was .24 and .22 , respectively $[t(39)=$ $0.98, S E=.02$, n.s.]. Although this is consistent with the interpretation mentioned above, note that this is a purely speculative and post hoc notion, and that a similar pattern did not emerge for Experiment 3 (rating 3, predictive $=$ .14 vs. nonpredictive $=.14$; rating 4 , predictive $=.26$ vs. nonpredictive $=.25) .{ }^{4}$

Importantly, we are not arguing against a general dualprocess account of recognition memory or even against a primarily retrieval-based conceptualization of the recollection process itself. Indeed, we find the large body of reported dissociations between recollection and familiarity quite convincing of this perspective. Rather, we are arguing that when it comes to gauging subjective states of awareness in memory, one cannot overlook the important role played by cognitive heuristics in general and by processing fluency in particular. We believe that the implications of the present study are most illuminated when viewed in light of the body of findings mentioned earlier that demonstrate reliable effects of processing fluency on various memorial and nonmemorial subjective judgments (see, e.g., Begg et al., 1996; Hasher et al., 1981; Hasher et al., 1977; Jacoby, Kelley et al., 1989; Jacoby, Woloshyn, \& Kelley, 1989; Mandler et al., 1987; Masson \& Caldwell, 1998; Reber et al., 1998; Reber et al., 2004; Westerman \& Greene, 1999). Regardless of whether the recollection process itself is best conceptualized as an all-or-none threshold retrieval mechanism (e.g., Yonelinas, 1994), we argue that claims of recollection should be viewed as a specific type of subjective judgment that-like those mentioned earlier - is based on multiple types of stimulus information (i.e., all available evidence), some of which is consciously retrieved and some of which is not.

Still, although we interpret the findings of Experiments 2 and 3 as being due to the widespread influence of processing fluency upon subjective judgments in general, it is possible that there is more at play here than what we have mentioned thus far. For instance, it is unclear from the present findings whether and to what extent participants' elevated reports of recollection to test items preceded by primes (Experiment 2) and predictive sentence stems (Experiment 3 ) were accompanied by illusory conscious feelings of prior presentation, a phenomenon that has been referred to as phantom recollection (see, e.g., Brainerd, Payne, Wright, \& Reyna, 2003). Because phantom recollection has been most extensively investigated from the perspective of fuzzy-trace theory (e.g., Brainerd \& Reyna, 1990), the methods that have been most often employed are those that manipulate semantic relatedness between targets and nonstudied lures, as in the Deese/RoedigerMcDermott paradigm (Deese, 1959; Roediger \& McDermott, 1995). As such, it is unknown what effect, if any, manipulations of processing fluency might have on phantom recollection. Interestingly, the illusory conscious feelings of prior presentation that accompany states of phantom recollection are so convincing that participants are actually willing to assign contextual detail to nonstudied lures (e.g., Payne, Elie, Blackwell, \& Neuschatz, 1996). The present study does not speak to such phenomena, and future studies will have to be conducted to determine the degree to which (if any) such illusory conscious feelings are responsible for our pattern of results.

For now, the present findings are taken - at the very least - as support for the contention that claims of remembering do not directly map onto an underlying thresholdlike retrieval mechanism. Rather, we see claims of remembering as yet another example of a particular type of subjective judgment that is partly inferential in nature and, as such, reliably influenced by the relative ease and speed with which stimulus information is processed.

\section{AUTHOR NOTE}

We are grateful to Erin Rabideau, Kathryn Carpenter, Brandon Ashinoff, Melissa Schlactus, Natalie Berlin, Max Levin, and Zachary Messer for their assistance in data collection. Correspondence concerning this article should be addressed to B. P. Kurilla, Department of Psychology, State University of New York, P.O. Box 6000, Binghamton, NY 13902 (e-mail: bkurill1@binghamton.edu).

\section{REFERENCES}

Atkinson, R., \& Juola, J. F. (1973). Factors influencing speed and accuracy of word recognition. In S. Kornblum (Ed.), Attention and performance IV (pp. 583-611). New York: Academic Press.

BegG, I. M., Robertson, R. K., Gruppuso, V., Anas, A., \& Needham, 
D. R. (1996). The illusory-knowledge effect. Journal of Memory \& Language, 35, 410-433.

BODNER, G. E., \& LindSAY, D. S. (2003). Remembering and knowing in context. Journal of Memory \& Language, 48, 563-580.

Brainerd, C. J., Payne, D. G., Wright, R., \& Reyna, V. F. (2003). Phantom recall. Journal of Memory \& Language, 48, 445-467.

Brainerd, C. J., \& Reyna, V. F. (1990). Gist is the grist: Fuzzy-trace theory and the new intuitionism. Developmental Review, 10, 3-47.

CARY, M., \& Reder, L. M. (2003). A dual-process account of the listlength and strength-based mirror effects in recognition. Journal of Memory \& Language, 49, 231-248.

DEESE, J. (1959). On the prediction of occurrence of particular verbal intrusions in immediate recall. Journal of Experimental Psychology, 58, 17-22.

GARDINER, J. M. (1988). Functional aspects of recollective experience. Memory \& Cognition, 16, 309-313.

Gardiner, J. M., GregG, V. H., \& Karayianni, I. (2006). Recognition memory awareness: Occurrence of perceptual effects in remembering or in knowing depends on conscious resources at encoding, but not at retrieval. Memory \& Cognition, 34, 227-239.

Gardiner, J. M., Java, R., \& Richardson-Klavehn, A. (1996). How level of processing really influences awareness in recognition memory. Canadian Journal of Experimental Psychology, 50, 114-122.

Gruppuso, V., Lindsay, D. S., \& Kelley, C. M. (1997). The process dissociation procedure and similarity: Defining and estimating recollection and familiarity in recognition memory. Journal of Experimental Psychology: Learning, Memory, \& Cognition, 23, 259-278.

Hasher, L., Attig, M. S., \& Alba, J. W. (1981). I knew it all along, or did I? Journal of Verbal Learning \& Verbal Behavior, 20, 86-96.

Hasher, L., Goldstein, D., \& Toppino, T. (1977). Frequency and the conference of referential validity. Journal of Verbal Learning \& Verbal Behavior, 16, 107-112.

Higham, P. A., \& VoKey, J. R. (2004). Illusory recollection and dualprocess models of recognition memory. Quarterly Journal of Experimental Psychology, 57A, 714-744.

Hintzman, D. L., \& CurRan, T. (1994). Retrieval dynamics of recognition and frequency judgments: Evidence for separate processes of familiarity and recall. Journal of Memory \& Language, 33, 1-18.

Hirshman, E., \& Henzler, A. (1998). The role of decision processes in conscious recollection. Psychological Science, 9, 61-65.

JACOBY, L. L. (1991). A process dissociation framework: Separating automatic from intentional uses of memory. Journal of Memory \& Language, 30, 513-541.

JACOBY, L. L., \& Dallas, M. (1981). On the relationship between autobiographical memory and perceptual learning. Journal of Experimental Psychology: General, 110, 306-340.

Jacoby, L. L., Kelley, C. M., Brown, J., \& Jasechko, J. (1989). Becoming famous overnight: Limits on the ability to avoid unconscious influences of the past. Journal of Personality \& Social Psychology, 56, 326-338.

Jacoby, L. L., Kelley, C. M., \& Dywan, J. (1989). Memory attributions. In H. L. Roediger III \& F. I. M. Craik (Eds.), Varieties of memory and consciousness: Essays in honour of Endel Tulving (pp. 391-422). Hillsdale, NJ: Erlbaum.

JACOBY, L. L., \& Whitehouse, K. (1989). An illusion of memory: False recognition influenced by unconscious perception. Journal of Experimental Psychology: General, 118, 126-135.

Jacoby, L. L., Woloshyn, V., \& Kelley, C. M. (1989). Becoming famous without being recognized: Unconscious influences of memory provided by dividing attention. Journal of Experimental Psychology: General, 118, 115-125.

Kelley, C. M., \& Rhodes, M. G. (2002). Making sense and nonsense of experience: Attributions in memory and judgment. In B. H. Ross (Ed.), Psychology of learning and motivation: Advances in theory and research (Vol. 41, pp. 293-320). San Diego: Academic Press.

Leboe, J. P., \& Whittlesea, B. W. A. (2002). The inferential basis of familiarity and recall: Evidence for a common underlying process. Journal of Memory \& Language, 46, 804-829.

Lindsay, D. S., \& Kelley, C. M. (1996). Creating illusions of familiarity in a cued recall remember/know paradigm. Journal of Memory \& Language, 35, 197-211.

MandLer, G. (1980). Recognizing: The judgment of previous occurrence. Psychological Review, 87, 252-271.
Mandler, G., Nakamura, Y., \& Van Zandt, B. J. (1987). Nonspecific effects of exposure on stimuli that cannot be recognized. Journal of Experimental Psychology: Learning, Memory, \& Cognition, 13, 646-648.

Masson, M. E. J., \& Caldwell, J. I. (1998). Conceptually driven encoding episodes create perceptual misattributions. Acta Psychologica, 98, 183-210.

Miller, J. K., Lloyd, M. E., \& Westerman, D. L. (2007). When modality matters: Perceptual versus conceptual fluency-based illusions in recognition memory. Manuscript submitted for publication.

Payne, D. G., Elie, C. J., Blackwell, J. M., \& Neuschatz, J. S. (1996). Memory illusions: Recalling, recognizing, and recollecting events that never occurred. Journal of Memory \& Language, 35, 261-285.

RAJARAM, S. (1993). Remembering and knowing: Two means of access to the personal past. Memory \& Cognition, 21, 89-102.

Rajaram, S. (1996). Perceptual effects on remembering: Recollective processes in picture recognition memory. Journal of Experimental Psychology: Learning, Memory, \& Cognition, 22, 365-377.

Reber, R., Winkielman, P., \& SchWARZ, N. (1998). Effects of perceptual fluency on affective judgments. Psychological Science, 9, 45-48.

Reber, R., Zimmermann, T. D., \& Wurtz, P. (2004). Judgments of duration, figure-ground contrast, and size for words and nonwords. Perception \& Psychophysics, 66, 1105-1114.

Reder, L. M., Nhouyvanisvong, A., Schunn, C. D., Ayers, M. S., Angstadt, P., \& Hiraki, K. (2000). A mechanistic account of the mirror effect for word frequency: A computational model of remember-know judgments in a continuous recognition paradigm. Journal of Experimental Psychology: Learning, Memory, \& Cognition, 26, 294-320.

Roediger, H. L., III, \& McDermott, K. B. (1995). Creating false memories: Remembering words not presented in lists. Journal of Experimental Psychology: Learning, Memory, \& Cognition, 21, 803-814.

Rotello, C. M., Macmillan, N. A., \& Reeder, J. A. (2004). Sumdifference theory of remembering and knowing: A two-dimensional signal-detection model. Psychological Review, 111, 588-616.

Rotello, C. M., Macmillan, N. A., Reeder, J. A., \& Wong, M. (2005). The remember response: Subject to bias, graded, and not a process-pure indicator of recollection. Psychonomic Bulletin \& Review, 12, 865-873.

Tulving, E. (1985). Memory and consciousness. Canadian Psychology, 26, 1-12.

TVersky, A., \& Kahneman, D. (1974). Judgment under uncertainty: Heuristics and biases. Science, 185, 1124-1131.

Westerman, D. L. (2001). The role of familiarity in item recognition, associative recognition, and plurality recognition on self-paced and speeded tests. Journal of Experimental Psychology: Learning, Memory, \& Cognition, 27, 723-732.

Westerman, D. L., \& Greene, R. L. (1999). The effects of recent exposure on general-knowledge estimation. Memory, 7, 197-208.

Westerman, D. L., Lloyd, M. E., \& Miller, J. K. (2002). The attribution of perceptual fluency in recognition memory: The role of expectation. Journal of Memory \& Language, 47, 607-617.

Whittlesea, B. W. A. (1993). Illusions of familiarity. Journal of Experimental Psychology: Learning, Memory, \& Cognition, 19, $1235-1253$.

Whittlesea, B. W. A. (1997). Production, evaluation and preservation of experiences: Constructive processing in remembering and performance tasks. In D. L. Medin (Ed.), The psychology of learning and motivation (Vol. 37, pp. 211-264). New York: Academic Press.

Whittlesea, B. W. A. (2002). Two routes to remembering (and another to remembering not). Journal of Experimental Psychology: General, 131, 325-348.

Whittlesea, B. W. A., \& Williams, L. D. (1998). Why do strangers feel familiar, but friends don't? A discrepancy-attribution account of feelings of familiarity. Acta Psychologica, 98, 141-165.

Whittlesea, B. W. A., \& Williams, L. D. (2000). The source of feelings of familiarity: The discrepancy-attribution hypothesis. Journal of Experimental Psychology: Learning, Memory, \& Cognition, 26, 547-565.

Whittlesea, B. W. A., \& Williams, L. D. (2001a). The discrepancyattribution hypothesis: I. The heuristic basis of feelings of familiarity. Journal of Experimental Psychology: Learning, Memory, \& Cognition, 27, 3-13. 
Whittlesea, B. W. A., \& Williams, L. D. (2001b). The discrepancyattribution hypothesis: II. Expectation, uncertainty, surprise, and feelings of familiarity. Journal of Experimental Psychology: Learning, Memory, \& Cognition, 27, 14-33.

WIXTED, J. T. (2007). Dual-process theory and signal-detection theory of recognition memory. Psychological Review, 114, 152-176.

Wixted, J. T., \& Stretch, V. (2004). In defense of the signal detection interpretation of remember/know judgments. Psychonomic Bulletin \& Review, 11, 616-641.

YonelinAS, A. P. (1994). Receiver-operating characteristics in recognition memory: Evidence for a dual-process model. Journal of Experimental Psychology: Learning, Memory, \& Cognition, 20, 1341-1354.

Yonelinas, A. P. (2002). The nature of recollection and familiarity: A review of 30 years of research. Journal of Memory \& Language, 46, 441-517.

YonelinAS, A. P., \& JACOBY, L. L. (1995). The relation between remembering and knowing as bases for recognition: Effects of size congruency. Journal of Memory \& Language, 34, 622-643.

\section{NOTES}

1. When Higham and Vokey (2004) used the independent scales method in conjunction with a procedure in which test words were preceded by primes of different durations, they observed a significant el- evation in the ratings of both familiarity and recollection for test words preceded by long duration primes in comparison with those preceded by short duration primes. However, the assumptions concerning the role of a preceding prime during test differ between our experiments and those of Higham and Vokey; thus, no direct comparison is being made between our results and theirs.

2. This criterion was selected with the expectation that there would likely be errors for the scenario that depicted strong recollection in the absence of familiarity, because this seemed to us to be the most unlikely of the four possible combinations. Surprisingly, however, errors were most common for the scenario depicting high familiarity in the absence of recollection (30\% of participants in Experiment 2 and 29\% in Experiment 3). Note, however, that the order of scenarios was not counterbalanced across participants and, as a result, this scenario always occurred first. Furthermore, because there were only four scenarios (one for each combination of high and low recollection and familiarity) it is not improbable that a correct answer might have occurred to some participants after the fact and, as such, that accuracy might have increased as the number of plausible choice alternatives decreased.

3. We thank Phil Higham for suggesting this possibility.

4. The data from 6 participants were lost prior to calculating these averages.

(Manuscript received November 3, 2006; revision accepted for publication May 30, 2007.) 